\title{
Disturbance of the Front Region of Magnetic Reconnection Outflow Jets due to the Lower-Hybrid Drift Instability
}

\author{
T. K. M. Nakamura $\odot,{ }^{1, *}$ T. Umeda $\odot,{ }^{2}$ R. Nakamura, ${ }^{1}$ H. S. Fu $\odot,{ }^{3}$ and M. Oka $\odot^{4}$ \\ ${ }^{1}$ Space Research Institute, Austrian Academy of Sciences, Graz 8042, Austria \\ ${ }^{2}$ Institute for Space-Earth Environmental Research, Nagoya University, Nagoya 464-8601, Japan \\ ${ }^{3}$ School of Space and Environment, Beihang University, Beijing 100083, China \\ ${ }^{4}$ Space Sciences Laboratory, University of California, Berkeley, California 94720, USA
}

(Received 9 April 2019; revised manuscript received 2 September 2019; published 2 December 2019)

\begin{abstract}
A 3D fully kinetic simulation shows that the lower-hybrid drift instability disturbs the front of magnetic reconnection outflow jets and additionally causes energy dissipation. The result is very consistent with a disturbance observed at the dipolarization front (DF) in Earth's magnetotail by the Magnetospheric Multiscale (MMS) mission. A fully kinetic dispersion relation solver, validated by the MMS observations, further predicts that the disturbance of the reconnection jet front could occur over different parameter regimes in space plasmas including Earth's DF and solar flares.
\end{abstract}

DOI: $10.1103 /$ PhysRevLett.123.235101

Introduction.-Magnetic reconnection is a key process in collisionless plasmas that converts magnetic energy to plasma kinetic energies through changes in the magnetic field topology. The energy conversion in this process is believed to cause various explosive phenomena in space such as auroral substorms in Earth's magnetosphere and solar flares [1,2]. The topology change during reconnection occurs in a small-scale region, where plasmas are decoupled from the magnetic field. This so-called diffusion region is known to have a multiscale structure based on the ion and electron scales [3]. Ion and electron outflow jets are accelerated near the outer and inner diffusion regions, called the ion and electron diffusion regions, respectively [4]. Ions are known to be heated widely within the ion outflow jets [5]. Electrons are also heated near the electron diffusion region (EDR), where the energy dissipation term in the electron rest frame $\boldsymbol{J} \cdot \boldsymbol{E}^{\prime}=\boldsymbol{J} \cdot\left(\boldsymbol{E}+\boldsymbol{U}_{e} \times \boldsymbol{B}\right)$ is positive [6,7]. Here $\boldsymbol{J}, \boldsymbol{U}_{e}, \boldsymbol{E}$, and $\boldsymbol{B}$ are the current density, electron bulk velocity, and electric and magnetic fields, respectively.

Recent 2D and 3D kinetic simulations demonstrated that additional energy conversions take place even near the edges of the reconnection layer such as the reconnection separatrix, where inflow and outflow components are mixed, and the downstream side of the outflow jet fronts, where the reconnected field lines are strongly piled-up and sharp density and pressure gradient layers are formed [8,9].

Published by the American Physical Society under the terms of the Creative Commons Attribution 4.0 International license. Further distribution of this work must maintain attribution to the author(s) and the published article's title, journal citation, and DOI.
Recent 3D kinetic simulations demonstrated that the current perpendicular to the piled-up field at the strong density gradient drives the lower-hybrid drift instability (LHDI) [10-12], while the pressure gradient drives the ballooning-interchange instability [10,13]. A 3D fully kinetic simulation with a sufficiently large system size and high ion-to-electron mass ratio revealed that these instabilities driven at the jet fronts can actually couple to each other [10].

From an observational point of view, strong fluctuations in the electric field component perpendicular to the magnetic field have indeed been observed in situ by the Cluster spacecraft at jet fronts in Earth's magnetotail [commonly referred to as dipolarization fronts (DFs)], and they can be interpreted as being driven by LHDI [14]. In addition, in situ higher-time-resolution measurements by the Magnetospheric Multiscale (MMS) mission successfully confirmed the occurrence of the additional energy dissipation by the perpendicular electric field fluctuations at DFs [15]. However, the precise condition of the onset of these turbulent fluctuations at the jet fronts remains unclear. Therefore, in this paper, we combine results from a 3D fully kinetic simulation, MMS observations, and linear analyses in order to quantitatively discuss the onset condition of the LHDI turbulence.

Simulation settings. - A 3D fully kinetic simulation using the particle-in-cell code EM3D [16] is performed under nearly the same settings as the ones in Ref. [10]. The magnetic field and density profiles are set up as $B_{x}(z)=B_{b} \tanh \left(z / D_{0}\right)$ (Harris sheet) and $n(z)=n_{0} \operatorname{sech}^{2}\left(z / D_{0}\right)+n_{b}$, where $D_{0}$ is the half thickness of the initial sheet, and $n_{0}$ and $n_{b}$ are the Harris and background density components, respectively. The half thickness $D_{0}$ is set to be $0.8 d_{i}$, where $d_{i}$ is the ion inertial length based on $n_{0}$. The density ratio $n_{b} / n_{0}=0.05$, 


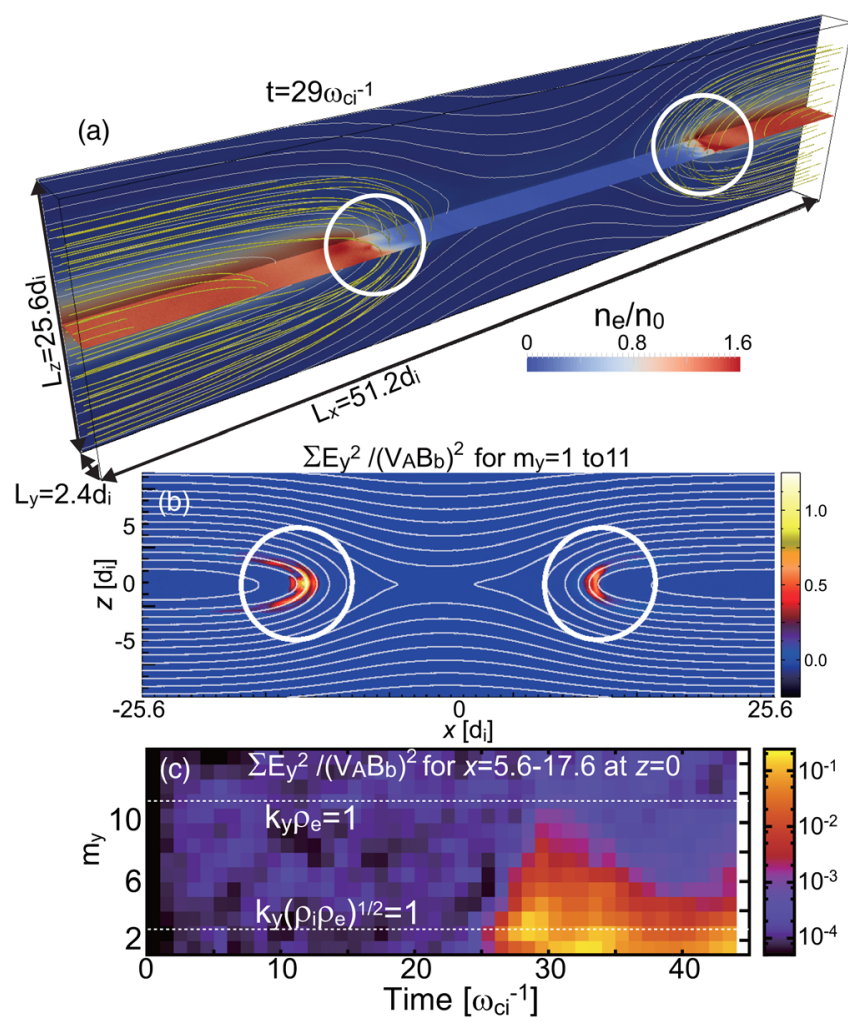

FIG. 1. (a) A 3D view of magnetic field lines at $t=29 \omega_{c i}^{-1}$ near the reconnection jet fronts with the $2 \mathrm{D}$ contours in the $x-z$ plane at $y=0$ and in the $x-y$ plane at $z=0$ of electron density $n_{e} . \omega_{c i}$ is the ion gyro frequency based on $B_{b}$. (b) Integrated amplitudes at each $(x, z)$ grid point of $k_{y}$ modes for $E_{y} /\left(V_{A} B_{b}\right)$ in the range $m_{y}=1-11$ at $t=29 \omega_{c i}^{-1} . V_{A}$ is the Alfvén speed based on $n_{0}$ and $B_{b}$. (c) Time evolutions of integrated amplitudes of $k_{y}$ modes for $E_{y}$ at $x=5.6-17.6$ and $z=0$.

the ion-to-electron temperature ratio $T_{i} / T_{e}=2.5$, and the ion-to-electron mass ratio $m_{i} / m_{e}=256$ are used. The ratio between the electron plasma frequency and the gyro frequency is $\omega_{p e} / \omega_{c e}=1.25$. The system size is $L_{x} \times L_{y} \times$ $L_{z}=51.2 \times 2.4 \times 25.6 \mathrm{di}^{3}=2048 \times 96 \times 1024$ cells with 100 particles per cell on average for each species. The system is periodic along the $x$ and $y$ directions, with conducting walls along the $z$ direction. To expedite the reconnection onset, a weak initial magnetic field perturbation is added according to $\delta \boldsymbol{B}=\boldsymbol{z} \times \nabla \psi$, where $\psi(x, z)=0.05$ $\cos \left(2 \pi x / L_{x}\right) \cos \left(\pi z / L_{z}\right)$.

Simulation results.-As reconnection develops, more magnetic field lines are piled up in the $x-z$ plane and larger density gradients in the $x$ direction are formed at the fronts of the reconnection outflow jets, as seen in Fig. 1(a). The perpendicular $\left(E_{y}\right)$ electric field fluctuations induced by the LHDI are seen in these density gradient layers as marked in Fig. 1(b) and previously reported by Nakamura et al. [10]. These fluctuations include the modes spreading from the hybrid $\left[\sim k_{y}\left(\rho_{i} \rho_{e}\right)^{1 / 2}\right]$ to electron $\left(\sim k_{y} \rho_{e}\right)$ kinetic scales [Fig. 1(c)], as predicted by a past linear analysis for
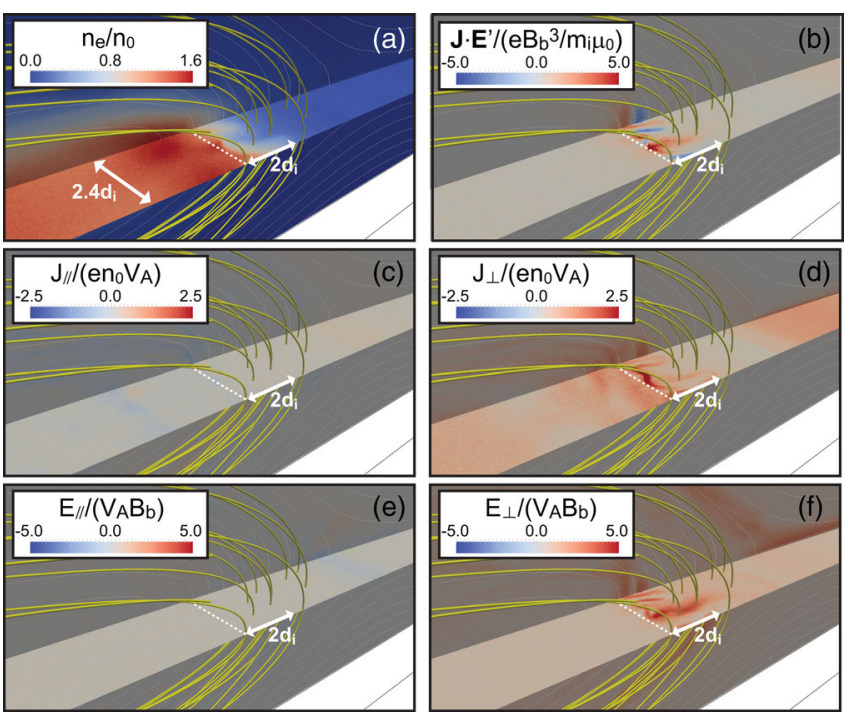

FIG. 2. Enlarged views near the reconnection jet fronts with the 2D contours of (a) the electron density $n_{e}$, (b) energy dissipation term $\boldsymbol{J} \cdot \boldsymbol{E}^{\prime}$, and (c)-(f) parallel and perpendicular components of the current density $\left(J_{\|}, J_{\perp}\right)$ and the electric field $\left(E_{\|}, E_{\perp}\right)$. The dashed lines indicate the edge of the high-density region.

the LHDI [17]. The growth rate of these $E_{y}$ modes are very consistent with the result from the present linear analysis for the LHDI, as will be shown in more detail later.

Figure 2 shows enlarged views of the reconnection jet front region, demonstrating how the LHDI appears in the simulation and contributes to the energy dissipation $\boldsymbol{J} \cdot \boldsymbol{E}^{\prime}$. The panels show strong fluctuations in both plasma and field components. Particularly, the significant fluctuations in the perpendicular components of the current density and electric field are seen near the density gradient layer. On the current density, the negative value of the parallel component is seen mainly on the high-density side, but the amplitude is much weaker than the perpendicular component $\left(\left|J_{\|}\right|<\left|J_{\perp}\right|\right)$ [see Figs. 2(c) and 2(d)]. The strong $J_{\perp}$ fluctuation is seen widely around the density gradient layer, with its peak located within the layer [see Fig. 2(d)]. On the electric field, the parallel component is negligible compared to the perpendicular component $\left(\left|E_{\|}\right| \ll\left|E_{\perp}\right|\right)$ [see Figs. 2(e) and 2(f)]. The strong $E_{\perp}$ fluctuation is seen locally within the density gradient layer [see Fig. 2(f)]. Thus, the strong $\boldsymbol{J} \cdot \boldsymbol{E}^{\prime}$ fluctuation that is dominantly sustained by $J_{\perp} E_{\perp}$ is seen within the density gradient layer.

The left panels in Fig. 3 show the averaged values of selected plasma and field components along the $x$ direction in the $z=0$ plane. Figures 3(a) and 3(b) show the piled-up field and the corresponding formation of the density gradient layer. The blue curve in Fig. 3(c), which corresponds to the amplitude of the $E_{y}$ fluctuation in the $y$ direction, shows that the fluctuation is enhanced within the gradient layer, indicating the evolution of the LHDI. Corresponding to the $E_{y}\left(\sim E_{\perp}\right)$ variation, the strong variation of $\boldsymbol{J} \cdot \boldsymbol{E}^{\prime}$ is seen within the gradient layer. The average 

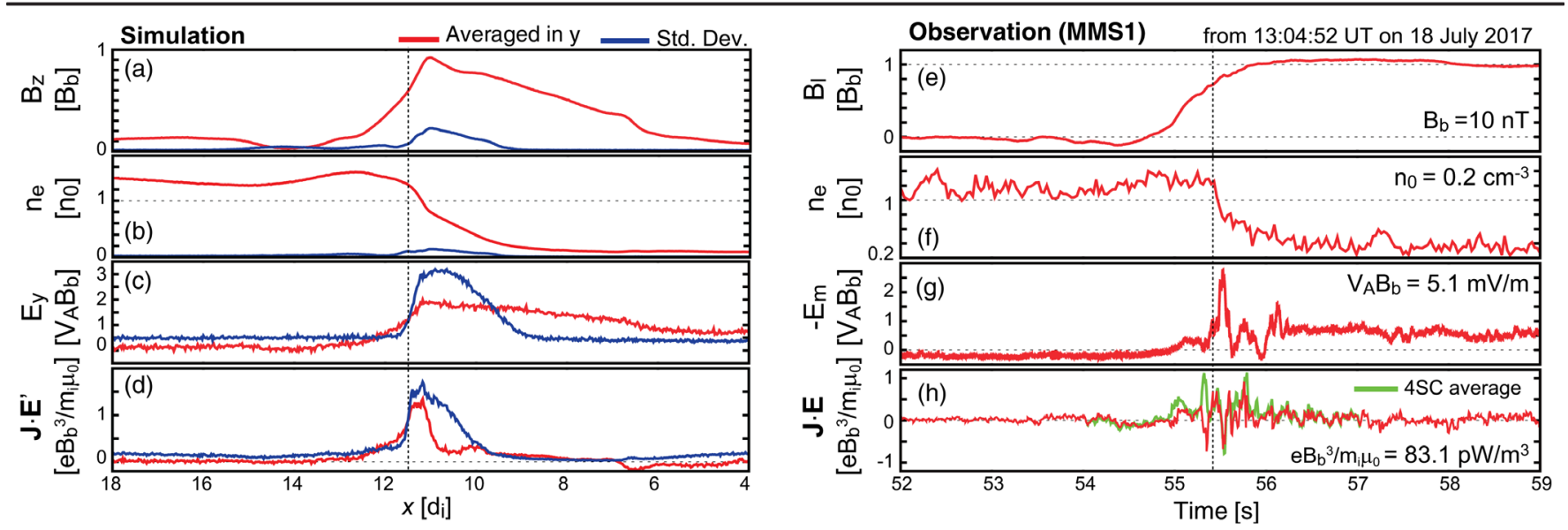

FIG. 3. Values along the $x$ direction at $z=0$ averaged in the $y$ direction near the reconnection jet fronts (left panels) and the in situ observations by the MMS1 spacecraft for $7 \mathrm{~s}$ from 13:04:52 UT on July 18, 2017 (right panels), of (a),(e) the $z$ ( $l$ ) component of the magnetic field $B_{z}\left(B_{l}\right)$, (b),(f) electron density $n_{e}$, (c),(g) y $(m)$ component of the electric field $E_{y}\left(-E_{m}\right)$, and (d), (h) dissipation term. The blue curves in (a)-(d) show the standard deviations. The green curve in (h) shows the averaged value for four MMS spacecraft. Here $l$ and $m$ components in (e) and (g) are the parallel and perpendicular components to the ambient magnetic field used by Liu et al. [15], which corresponds to $z$ and $y$ in the simulation. The dashed lines indicate the edges of the high-density intervals.

value of the $\boldsymbol{J} \cdot \boldsymbol{E}^{\prime}$ variation is positive, which means that the magnetic energy is additionally dissipated at the reconnection jet fronts. The peak value of the averaged $\boldsymbol{J} \cdot \boldsymbol{E}^{\prime} \quad\left(\sim 1\right.$ in the unit normalized by $m_{i}$ and $\left.B_{b}\right)$ is comparable to or larger than the values seen near the EDR in past kinetic simulations [5]. This indicates that the LHDI turbulence has a substantial effect on the energetics of reconnection.

Comparison with the MMS observations.-Interestingly, the above detailed features of the LHDI turbulence at the reconnection jet fronts are very consistent with the observed features at the DF in Earth's magnetotail on July 18, 2017, which were reported in Liu et al. [15]. In this event, the high-time-resolution (30-ms) MMS spacecraft [18] crossed the typical DF structures, which can be interpreted as being formed by the reconnection outflow jets, at around 13:04:55 universal time (UT). During the DF crossing, the four MMS spacecraft were located at around $[-22.9,-2.74,5.15]$ Earth radii $\left(R_{E}\right)$ in geocentric-solar-magnetospheric coordinates with their separations around $10 \mathrm{~km}$ in average. As partially shown in the right panels of Fig. 3, which were obtained by modifying the plots from Liu et al. [15], smallscale density and field fluctuations were seen within the density gradient layer, as seen in the present simulation. First, from the time interval of the crossing of the gradient layer ( 1.5 s), Liu et al. [15] estimated the layer thickness as the order of $600 \mathrm{~km} \sim d_{i}$, which is consistent with the present simulation. On the electric field structure, Liu et al. [15] reported that $E_{\|}$was negligible, while the strong $E_{\perp}$ fluctuation was seen locally within the layer. As shown in Fig. $3(\mathrm{~g})$, the peak amplitude $(\sim 15 \mathrm{mV} / \mathrm{m})$ of the $E_{\perp}$ $\left(\sim E_{m}\right)$ fluctuation is estimated as $\sim 3 V_{A} B_{b}$ based on the observed values, where $n \sim 0.2 \mathrm{~cm}^{-3}$ and $B_{b} \sim 10 \mathrm{nT}$ $\left(V_{A} \sim 510 \mathrm{~km} / \mathrm{s}\right)$, assuming that $B_{b}$ is close to the peak value near the DF. On the current density, Liu et al. [15] reported that the weak negative $J_{\|}$was seen on the high-density side of the density gradient layer, while the strong $J_{\perp}$ fluctuation was seen widely around the gradient layer, with its peak located within the layer. These field fluctuations result in the strong $\boldsymbol{J} \cdot \boldsymbol{E}\left(\sim J_{\perp} E_{\perp}\right)$ fluctuation within the gradient layer, whose amplitude was positive on average $\left(\sim 100 \mathrm{pW} / \mathrm{m}^{3} \sim 1 e B_{b}^{3} / m_{i} \mu_{0}\right.$ for $\left.B_{b} \sim 10 \mathrm{nT}\right)$, as shown in Fig. 3(h). These variation patterns as well as the normalized amplitudes of the field fluctuations are quantitatively consistent with the present simulation (compare the left and right panels of Fig. 3). Note that the dissipation term in the observations is in the spacecraft frame $(\boldsymbol{J} \cdot \boldsymbol{E})$, while that in the simulation is in the electron frame $\left[\boldsymbol{J} \cdot \boldsymbol{E}^{\prime}=\right.$ $\left.\boldsymbol{J} \cdot\left(\boldsymbol{E}+\boldsymbol{U}_{e} \times \boldsymbol{B}\right)\right]$. However, since the current is carried mainly by electrons near the gradient layer in both simulations and observations, $\boldsymbol{J} \cdot \boldsymbol{E}$ would be close to $\boldsymbol{J} \cdot \boldsymbol{E}^{\prime}$.

Linear analysis.-To understand the onset condition of the LHDI-driven turbulence at the reconnection jet fronts, a linear dispersion relation solver for fully kinetic plasma with a perpendicular drift, which was recently developed by Umeda and Nakamura [19], is applied to the present simulation and the MMS observations. The dispersion relation is derived in the electromagnetic and fully kinetic regime for ions and electrons that drift in the direction perpendicular to the magnetic field (see Ref. [19] for more details about this linear dispersion relation). The dispersion solver requires $m_{i} / m_{e}, T_{i} / T_{e}, \omega_{p e} / \omega_{c e}$, the ion and electron drift velocities relative to the thermal speeds $V_{d} / V_{t}$, and the ratio between the thermal speeds and the speed of light $V_{t} / c$ for ions and electrons as input parameters to obtain the dispersion relation.

Figure 4(a) shows the results of the linear analysis for the present simulation. The input parameters, which are 

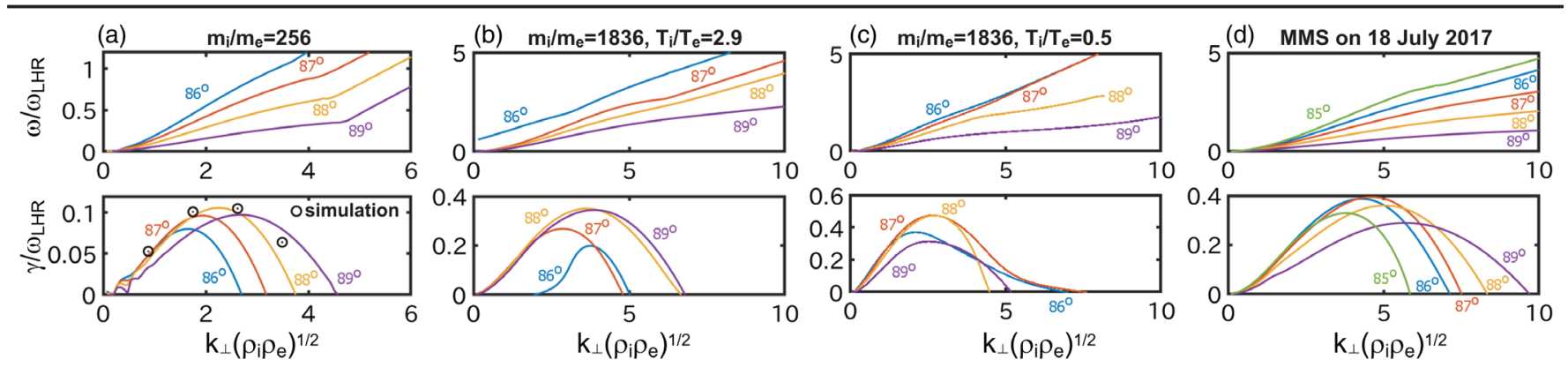

FIG. 4. Linear dispersion relations at the jet fronts derived by Umeda and Nakamura [19]. Panels show wave modes with wave normal angles quasiperpendicular to the ambient magnetic field $\left(\phi=85^{\circ}-89^{\circ}\right)$ for parameters (a) obtained from the simulation, (b) similar to (a) but for the real ion-to-electron mass ratio, (c) the same as (b) but for $T_{i} / T_{e}=0.5$, and (d) obtained from the MMS observations on July 18, 2017 [15].

obtained as local values near the $\boldsymbol{J} \cdot \boldsymbol{E}^{\prime}$ peak shown in Fig. 3(d) (at $x \sim 11.2 d_{i}$ ), are $m_{i} / m_{e}=256, T_{i} / T_{e}=2.6$, $\omega_{p e} / \omega_{c e}=1.8, V_{d i} / V_{t i}=0.30, V_{d e} / V_{t e}=0.06\left(\mid V_{d i}-\right.$ $\left.V_{d e} \mid \sim 0.09 V_{t e} \sim 0.79 V_{A}\right), \quad V_{t i} / c=0.030$, and $V_{t e} / c=$ $0.30(\beta \sim 2.12)$. The dispersion relation demonstrates that a quasiperpendicular wave mode with a wave normal angle of $\phi \sim 88^{\circ}$ relative to the ambient magnetic field has the maximum growth rate. The quasiperpendicular wave modes with normal angles around $88^{\circ}$ have a large growth rate of $\gamma \sim 0.1 \omega_{\mathrm{LHR}}$ at a wave number $k_{\perp}\left(\rho_{i} \rho_{e}\right)^{1 / 2} \sim 2$, where $\omega_{\mathrm{LHR}}$ is the lower-hybrid frequency. The black circles show the growth rates of the $E_{y}^{2}$ amplitudes obtained from the present simulation for $m_{y}=1$ to four modes. The obtained rates are in good agreements with the linear analysis. This clearly indicates that the $E_{y}$ fluctuations seen in the simulation are caused by the perpendicular plasma drifts and the related growth of the LHDI as confirmed by the linear analysis.

To investigate whether the LHDI occurs even under realistic conditions at the reconnection jet front regions, the linear analysis is also performed by modifying the above input parameters to the ones for realistic $m_{i} / m_{e}$ and $V_{t} / c$. The input parameters are $m_{i} / m_{e}=1836, T_{i} / T_{e}=2.9$, $\omega_{p e} / \omega_{c e}=16.4, V_{d i} / V_{t i}=0.75, V_{d e} / V_{t e}=0.06\left(\mid V_{d i}-\right.$ $\left.V_{d e} \mid \sim 0.09 V_{t e} \sim 2.10 V_{A}\right), \quad V_{t i} / c=0.001$, and $V_{t e} / c=$ $0.03(\beta \sim 2.34)$. Here $m_{i} / m_{e}$ is modified to the real mass ratio, and $V_{t e} / c$ is modified to the ratio between $V_{t e}$ and the real speed of light by assuming $V_{t e} \sim 10^{4} \mathrm{~km} / \mathrm{s}$ $\left(T_{e} \sim 1 \mathrm{keV}\right)$. To this end, the ion thermal speed relative to the electron thermal speed is adjusted to keep $T_{i} / T_{e}$ as a similar value to the present simulation $\left(T_{i} / T_{e} \sim 2.5-3\right)$, and the strength of the magnetic field (i.e., $\omega_{p e} / \omega_{c e}=$ $c / V_{A e}$ ) is adjusted to keep the plasma $\beta$ as a value similar to the simulation $(\beta \sim 2)$. Figure 4(b) shows the results for these modified parameters. The dispersion relation demonstrates that a quasiperpendicular wave mode with $\phi \sim 88^{\circ}$ relative to the ambient magnetic field has the maximum growth rate as in the above case in Fig. 4(a). The quasiperpendicular wave modes with normal angles around $88^{\circ}$ have large growth rates of $\gamma \sim 0.35 \omega_{\mathrm{LHR}}$, which is about 3-3.5 times larger than the above case, at a wave number $k_{\perp}\left(\rho_{i} \rho_{e}\right)^{1 / 2} \sim 3.5$, which is about 1.5-2 times larger than the above case. This indicates that the LHDI would occur easily even under realistic conditions with the realistic $m_{i} / m_{e}$. We also confirmed that these results are not significantly affected by $T_{i} / T_{e}$, as seen in Fig. 4(c), in which $T_{i} / T_{e}$ is set to 0.5 by modifying $V_{t i} / c$ with the same parameters as the case in Fig. 4(b).

Next, the linear analysis is performed under real parameters obtained from the MMS observation event on July 18, 2017. The input parameters, which are obtained as local values near the time interval with large amplitudes of the field fluctuations shown in Figs. 3(g) and 3(h) $\left(\sim 13: 04: 55.5\right.$ UT), are $m_{i} / m_{e}=1836, \quad T_{i} / T_{e}=4.6$, $\omega_{p e} / \omega_{c e}=20.0, V_{d i} / V_{t i}=0.10, V_{d e} / V_{t e}=0.10\left(\mid V_{d i}-\right.$ $\left.V_{d e} \mid \sim 0.11 V_{t e} \sim 3.00 V_{A}\right), \quad V_{t i} / c=0.002$, and $V_{t e} / c=$ $0.03(\beta \sim 4.97)$. Figure 4(d) shows the results of the linear analysis for this MMS event. The dispersion relation demonstrates that a quasiperpendicular wave mode with $\phi \sim 87^{\circ}$ relative to the ambient magnetic field has the maximum growth rate. The quasiperpendicular wave modes with normal angles around $87^{\circ}$ have a large growth rate of $\gamma \sim 0.4 \omega_{\mathrm{LHR}}$ at a wave number $k_{\perp}\left(\rho_{i} \rho_{e}\right)^{1 / 2} \sim 4.5$, both of which are close to the above cases under realistic parameters. The wave number of the fastest growing mode corresponds to $\lambda \sim 100 \mathrm{~km}$. The phase speed of the fastest mode, which is obtained from the slope of the upper panel of Fig. 4(d), is $v_{p h} \sim 1000 \mathrm{~km} / \mathrm{s}$. Thus, the estimated observation period of this LHDI wave is $\Delta t \sim 0.1 \mathrm{~s}$. This estimated $\Delta t$ is in excellent agreement with the observed periods for the field fluctuations in this MMS event [see Figs. 3(g) and 3(h)]. Together with the above quantitative consistencies between the simulation and the MMS event on the structures of plasma and field fluctuations near the density gradient layer, these results naturally suggest that the LHDI-driven turbulence and the resulting energy dissipation at the reconnection jet fronts as seen in the present simulation may really occur in Earth's magnetotail.

Discussions.-Based on the parameter range used in the present simulation and linear analysis, the LHDI turbulence 
would occur under conditions with a perpendicular drift speed close to $V_{d} \sim V_{A}$, a moderate $\beta(\sim 1-5)$ and a large $w_{p e} / w_{c e}(>1)$, not significantly depending on $T_{i} / T_{e}$. Assuming that the minimum thickness of the gradient layer in the reconnection jet front region is the order of $d_{i}$ as frequently observed at Earth's DFs [15,20,21], the perpendicular drift speed $V_{d}$ would be the order of $V_{A}$ based on the local piled-up field strength $B_{p}$, as, indeed, recently observed by MMS [22]. Assuming that the upstream plasma $\beta$ is sufficiently low, the plasma pressure in the jet front region $P_{f}$ is not significantly changed from the background value $P_{0}$, and the maximum $B_{p} \sim B_{b}$, as also frequently observed at Earth's DFs [23], $P_{f}$ should be balanced with $B_{b}^{2} / 2 \mu_{0}$, and hence the local $\beta$ in the jet front regions would be of the order $\beta \sim 1$ (i.e., $P_{f} \sim$ $\left.P_{0} \sim B_{b}^{2} / 2 \mu_{0} \sim B_{p}^{2} / 2 \mu_{0}\right)$. Thus, the conditions of $V_{d} \sim$ $V_{A}$ and $\beta \sim 1$ would generally be satisfied in the reconnection jet front regions when the process is driven by low- $\beta$ upstream plasma as observed in Earth's magnetotail lobes [24] and estimated in the solar coronal loops [25,26]. Then, $\omega_{p e} / \omega_{c e}$ is written as

$$
\frac{\omega_{p e}}{\omega_{c e}} \sim 3\left(\frac{n}{1 \mathrm{~cm}^{-3}}\right)^{1 / 2}\left(\frac{B}{100 \mathrm{nT}}\right)^{-1} .
$$

In Earth's magnetotail, for example, since the plasma density $n$ and the magnetic field strength $B$ are generally of the order of $1 \mathrm{~cm}^{-3}$ and $10 \mathrm{nT}$, respectively, $\omega_{p e} / \omega_{c e}$ would be of the order of 10 , which is consistent with the above MMS event. In a solar flare, since the typical $n$ and $B$ are roughly in the range $10^{8}-10^{10} \mathrm{~cm}^{-3}$ and $1-100 \mathrm{G}$ $\left(10^{5}-10^{7} \mathrm{nT}\right)$, respectively [2], $\omega_{p e} / \omega_{c e}$ would be of the order of 1-10. Past comparison studies have also discussed these similarities of $\omega_{p e} / \omega_{c e}\left(\propto V_{A}\right)$ and plasma $\beta$ between the magnetotail and solar flares [27-29]. Thus, the LHDI turbulence as seen in the present simulation may frequently occur in the reconnection jet front regions at many locations in space including Earth's magnetotail and solar flares. However, it is noted that the evolution of the LHDI would become more complicated when considering the guide (out-of-plane) field component [17]. On the other hand, the density asymmetry across the primary reconnection layer would drive the LHDI more widely within the reconnection layer [30,31]. Although this initial study on the stability of the jet front region focused only on the simple symmetric case without the guide field, more systematic studies considering these nonideal factors are required to be performed to more comprehensively understand the stability conditions of the reconnection jet front regions.

This work was supported by the Austrian Research Fund (FWF): I2016-N20 and the joint research program of the Institute for Space-Earth Environmental Research (ISEE), Nagoya University. The simulations were performed on the FX100 supercomputer system at the Information
Technology Center, Nagoya University, supported by the "Computational Joint Research Program (Collaborative Research Project on Computer Science with HighPerformance Computing)" at the ISEE, Nagoya University. A part of the simulation data was analyzed with resources at the Space Research Institute of the Austrian Academy of Sciences. The authors thank I. Shinohara for setting the simulation code. The MMS spacecraft data are available via the MMS Science Data Center (https://lasp.colorado.edu/mms/sdc/public/). T. U. was supported by MEXT/JSPS KAKENHI No. JP19H01868. M. O. was supported by NASA Grants No. 80NSSC18K1002 and No. 80NSSC18K1373 at UC Berkeley and NNH16AC60I at Los Alamos National Laboratory (LANL).

*takuma.nakamura@oeaw.ac.at

[1] J. W. Dungey, Phys. Rev. Lett. 6, 47 (1961).

[2] K. Shibata and T. Magara, Living Rev. Solar Phys. 8, 6 (2011).

[3] M. A. Shay, J. F. Drake, R. E. Denton, and D. Biskamp, J. Geophys. Res. 103, 9165 (1998).

[4] P. L. Pritchett, J. Geophys. Res. 106, 3783 (2001).

[5] C. C. Haggerty, M. A. Shay, C. T. McHugh, J. F. Drake, and T. D. Phan, Geophys. Res. Lett. 42, 9657 (2015).

[6] S. Zenitani, M. Hesse, A. Klimas, and M. Kuznetsova, Phys. Rev. Lett. 106, 195003 (2011).

[7] A. Le, J. Egedal, and W. Daughton, Phys. Plasmas 23, 102109 (2016).

[8] G. Lapenta, M. Goldman, D. Newman, S. Markidis, and A. Divin, Phys. Plasmas 21, 055702 (2014).

[9] M. Hesse, C. Norgen, P. Tenfjord, J. L. Burch, Y.-H. Liu, L.-J. Chen et al., Phys. Plasmas 25, 122902 (2018).

[10] T. K. M. Nakamura, R. Nakamura, W. Baumjohann, T. Umeda, and I. Shinohara, Geophys. Res. Lett. 43, 8356 (2016).

[11] A. E. Vapirev, G. Lapenta, A. Divin, S. Markidis, P. Henri, M. Goldman, and D. Newman, J. Geophys. Res. 118, 1435 (2013).

[12] A. Divin, Y. V. Khotyaintsev, A. Vaivads, M. André, S. Markidis, and G. Lapenta, J. Geophys. Res. 120, 2675 (2015).

[13] P. L. Pritchett and F. V. Coroniti, J. Geophys. Res. 115, A06301 (2010).

[14] A. Divin, Y. V. Khotyaintsev, A. Vaivads, and M. André, J. Geophys. Res. 120, 1124 (2015).

[15] C. M. Liu, H. S. Fu, Y. Xu, Y. V. Khotyaintsev, J. L. Burch, R. E. Ergun, D. G. Gershman, and R. B. Torbert, Geophys. Res. Lett. 45, 4628 (2018).

[16] M. Hoshino, J. Geophys. Res. 92, 7368 (1987).

[17] W. Daughton, Phys. Plasmas 10, 3103 (2003).

[18] J. L. Burch, T. E. Moore, R. B. Torbert, and B. L. Giles, Space Sci. Rev. 199, 5 (2016).

[19] T. Umeda and T. K. M. Nakamura, Phys. Plasmas 25, 102109 (2018).

[20] D. Schmid, M. Volwerk, R. Nakamura, W. Baumjohann, and M. Heyn, Ann. Geophys. 29, 1537 (2011). 
[21] H. S. Fu, Y. V. Khotyaintsev, A. Vaivads, M. André, and S. Y. Huang, Geophys. Res. Lett. 39, L06105 (2012).

[22] C. M. Liu, H. S. Fu, A. Vaivads, Y. V. Khotyaintsev, D. J. Gershman, K.-J. Hwang et al., Geophys. Res. Lett. 45, 556 (2018).

[23] A. Runov, V. Angelopoulos, X.-Z. Zhou, X.-J. Zhang, S. Li, F. Plaschke, and J. Bonnell, J. Geophys. Res. 116, A05216 (2011).

[24] W. Baumjohann, G. Paschmann, and H. Lühr, Geophys. Res. Lett. 17, 45 (1990).

[25] K. Iwai, K. Shibasaki, S. Nozawa, T. Takahashi, S. Sawada, J. Kitagawa, S. Miyawaki, and H. Kashiwagi, Earth Planets Space 66, 149 (2014).

[26] G. A. Gary, Sol. Phys. 203, 71 (2001).
[27] T. Terasawa, K. Shibata, and M. Scholer, Adv. Space Res. 26, 573 (2000)

[28] J. Lin, S. R. Cranmer, and C. J. Farrugia, J. Geophys. Res. 113, A11107 (2008).

[29] M. Oka, J. Birn, M. Battaglia, C. C. Chaston, S. M. Hatch, G. Livadiotis, S. Imada, Y. Miyoshi, M. Kuhar, F. Effenberger, E. Eriksson, Y. V. Khotyaintsev, and A. Retinò, Space Sci. Rev. 214, 82 (2018).

[30] L. Price, M. Swisdak, J. F. Drake, P. A. Cassak, J. T. Dahlin, and R. E. Ergun, Geophys. Res. Lett. 43, 6020 (2016).

[31] A. Le, W. Daughton, O. Ohia, L.-J. Chen, Y.-H. Liu, S. Wang, W. D. Nystrom, and R. Bird, Phys. Plasmas 25, 062103 (2018). 